\title{
Secondary sclerosing cholangitis: an emerging complication in critically ill COVID-19 patients
}

\author{
Philippe Meersseman 1,2, Joris Blondee ${ }^{3,4}$, Greet De Vlieger ${ }^{5,6}$, Schalk van der Merwe $e^{7,8}$ \\ and Diethard Monbaliu ${ }^{3,4^{*}}$ (D) on behalf of the Collaborators Leuven Liver Transplant program
}

(c) 2021 Springer-Verlag GmbH Germany, part of Springer Nature

The hallmark pneumonia of SARS-CoV-2 infection (coronavirus disease 2019, COVID-19) is often accompanied by important extra-pulmonary manifestations. Liver dysfunction occurs in up to $45 \%$ of patients and manifests predominantly as moderate transaminitis. Although the hepatic expression of angiotensin-converting-enzyme-2 (ACE2) receptor is largely restricted to cholangiocytes, reports of cholestatic injury have been rare [1].

In the first 12 weeks of the pandemic, 3/114 COVID19 patients admitted to our tertiary intensive care unit (ICU) developed a rapidly progressive cholestatic liver injury that persisted after the acute respiratory distress syndrome (ARDS) had resolved, and evolved to a condition reminiscent of secondary sclerosing cholangitis in critically ill patients (SSC-CIP), a rare but often fatal complication in patients receiving prolonged critical care [2]. During the same time period, a fourth patient with this condition was referred to our center (Fig. 1).

The patients were male, aged 48-68, and required prolonged mechanical ventilation, renal support, and veno-venous extracorporeal membrane oxygenation (VV-ECMO, supplementary Table 1). Magnetic resonance cholangiopancreatography (MRCP) showed focal strictures in intrahepatic bile ducts with intraluminal

\footnotetext{
*Correspondence: diethard.monbaliu@uzleuven.be

3 Department of Microbiology, Immunology and Transplantation, Laboratory of Abdominal Transplantation, KU Leuven, Herestraat 49, 3000 Leuven, Belgium

Full author information is available at the end of the article

Philippe Meersseman and Joris Blondeel contributed equally.

The members of the Collaborators Leuven Liver Transplant program are listed in acknowledgements.
}

sludge and casts, the radiological hallmark of SSC-CIP. Liver biopsies showed findings consistent with biliary obstruction, typical for SSC (supplementary Fig. 2). Patients 1 and 2 ultimately required liver transplantation because of refractory cholangitis with irreversible biliary damage: patient 1 is currently doing well but patient 2 died of post-transplant pneumonia and septic shock. Patient 3 experienced a milder form of SSC-CIP and is currently doing well, while patient 4 died as a result of a lethal hepatic haemorrhage.

With an estimated prevalence of 1/2000 (0.05\%) ICU admissions, SSC-CIP was remarkably frequent with $3 / 114$ ICU patients (2.6\%) over 3 months and represented $3 / 74(4.1 \%)$ of mechanically ventilated and $3 / 13(23.1 \%)$ of VV-ECMO-treated patients [3]. COVID-19-specific disease and treatment factors may have precipitated biliary ischemia and cholangiopathy, including varying degrees of hemodynamic instability, high positive endexpiratory pressures reducing hepatosplanchnic blood flow, drug-induced bile duct injury by sedatives such as ketamine, parenteral nutrition, and the exaggerated proinflammatory cytokine storm that interferes with the biliary epithelium's physiological defense against hydrophobic bile salts [2, 4].

Importantly, SARS-CoV-2 RNA and nucleo-capsid protein have been detected in the cholangiocytes and bile of patients with fatal COVID-19 pneumonia, suggesting that a direct cytopathic effect may occur [5]. Moreover, endothelialitis resulting in hypercoagulability and microthrombi deposition in the peribiliary vascular plexus may aggravate ischemia of the biliary epithelium.

This report aims to raise awareness about the risk for COVID-19 patients to develop severe cholestatic liver

\section{Springer}




\section{Patient 1}
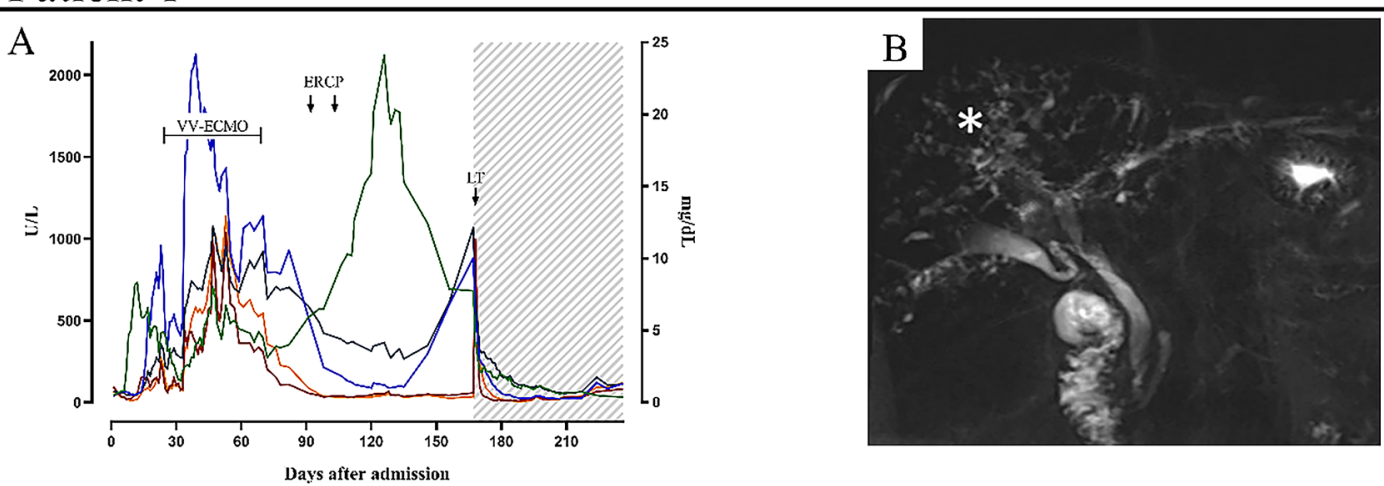

Patient 2
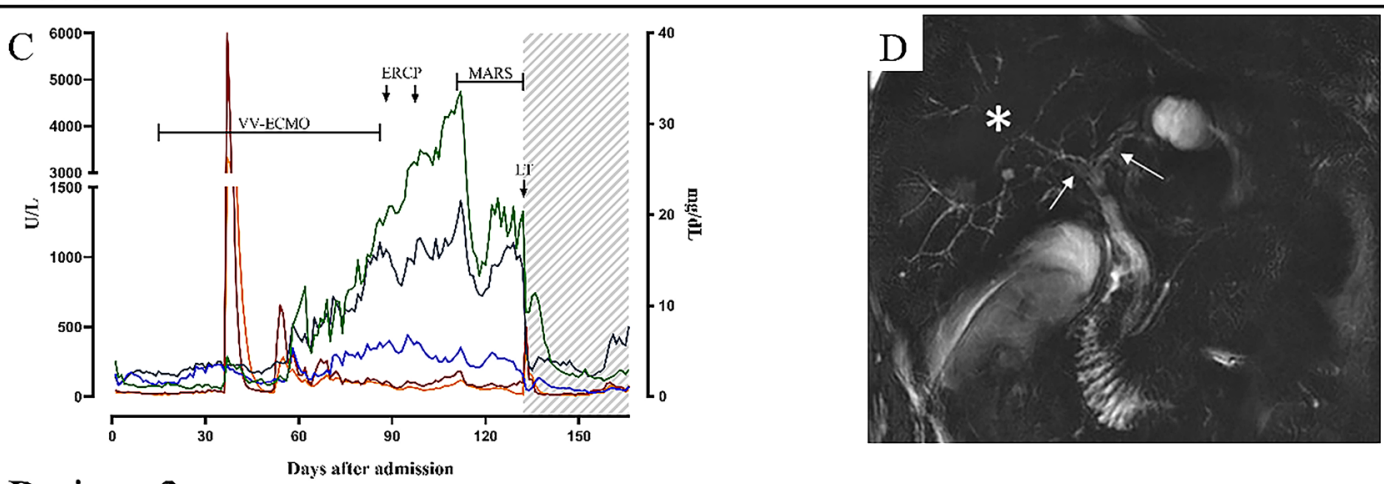

Patient 3
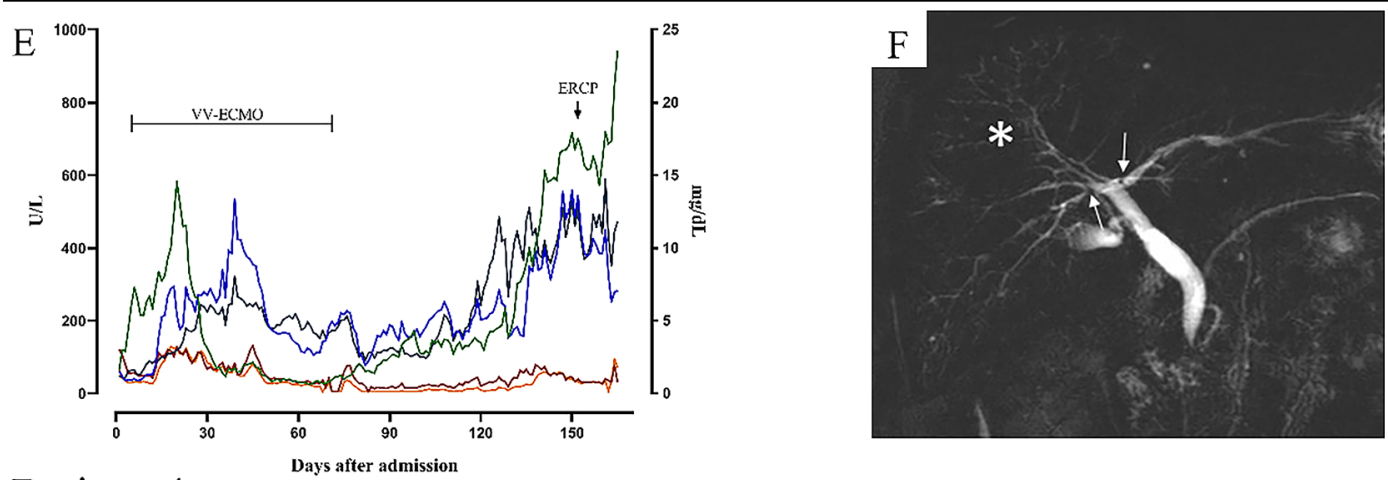

Patient 4
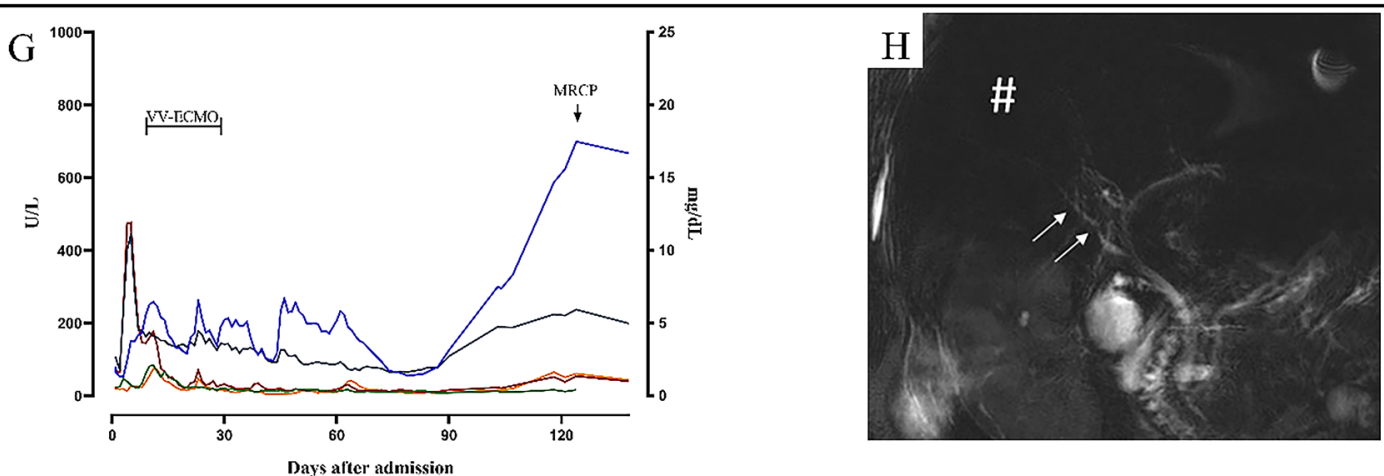

- Total bilirubine - Gamma GT - ALP

$$
\text { - AST - ALT }
$$


(See figure on previous page.)

Fig. 1 Temporal evolution of liver tests, treatments \& MRCP images. For each individual patient (rows), the temporal evolution of liver enzymes, along with time points when critical diagnostic or treatment events occurred (left). Levels of gamma-glutamyltransferase (Gamma GT), alkaline phosphatase (ALP), aspartate transaminase (AST) and alanine transaminase (ALT) are projected on the left vertical axis and expressed as U/L, total bilirubin is projected on the right vertical axis and is expressed as $\mathrm{mg} / \mathrm{dl}$. W-ECMO veno-venous extracorporeal membrane oxygenation, ERCP endoscopic retrograde cholangiopancreatography, MARS molecular absorbent recirculating system, LT liver transplantation. Cholestasis was defined as alkaline phosphatase > 1.5 times the upper limit. Also shown are a representative MRCP image for each patient (right) illustrating: in patient 1, diffuse beading of the intrahepatic biliary system $\left(^{*}\right)$; in patient 2 and 3, diffuse beading of the intrahepatic biliary ducts $\left(^{*}\right)$ and focal strictures on the left and right hepatic ducts (arrows); in patient 4, focal strictures on the right hepatic duct (arrows) and diminished arborisation of the intrahepatic biliary tree (\#). All these findings are consistent with the diagnosis of 'Secondary Sclerosing Cholangitis in Critically III Patients' (SSC-CIP)

dysfunction reminiscent of SSC-CIP. As COVID-19 becomes better understood, more patients may recover from ARDS and require prolonged critical care with its associated risks. Our data-although from a small cohort-indicate a spectrum of severity, ranging from asymptomatic bile duct abnormalities to cholangiosepsis. Infamous for its bleak prognosis, early diagnosis with MRCP is critical. Whether mild forms confer a risk for secondary biliary cirrhosis is unknown. The outcome of liver transplantation for COVID-19-cholangiopathy remains to be determined, but a timely multidisciplinary evaluation is warranted. A direct causal role of SARSCoV-2 in COVID-19-associated SSC-CIP is the subject of ongoing investigations.

\section{Supplementary Information}

The online version contains supplementary material available at https://doi. org/10.1007/s00134-021-06445-8.

\begin{abstract}
Author details
${ }^{1}$ Department of Microbiology, Immunology and Transplantation, Laboratory for Clinical Infectious and Inflammatory Disorders, KU Leuven, Leuven, Belgium. ${ }^{2}$ Department of General Internal Medicine, Medical Intensive Care Unit, University Hospitals Leuven, Leuven, Belgium. ${ }^{3}$ Department of Microbiology, Immunology and Transplantation, Laboratory of Abdominal Transplantation, KU Leuven, Herestraat 49, 3000 Leuven, Belgium. ${ }^{4}$ Department of Abdominal Transplant Surgery and Coordination, University Hospitals Leuven, Leuven, Belgium. ${ }^{5}$ Department of Cellular and Molecular Medicine, Laboratory of Intensive Care Medicine, KU Leuven, Leuven, Belgium. ${ }^{6}$ Department of Intensive Care Medicine, Intensive Care Unit, University Hospitals Leuven, Leuven, Belgium. ${ }^{7}$ Department of Chronic Diseases and Metabolism, Hepatology, KU Leuven, Leuven, Belgium. ${ }^{8}$ Department of Gastroenterology and Hepatology, University Hospitals Leuven, Leuven, Belgium.
\end{abstract}

\section{Acknowledgements}

Collaborators Leuven Liver Transplant program members are: Joost Wauters (Department of Microbiology, Immunology and Transplantation, Laboratory for Clinical Infectious and Inflammatory Disorders, KU Leuven, Leuven, Belgium; Department of General Internal Medicine, Medical Intensive Care Unit, University Hospitals Leuven, Leuven, Belgium), Alexander Wilmer (Department of Microbiology, Immunology and Transplantation, Laboratory for Clinical Infectious and Inflammatory Disorders, KU Leuven, Leuven, Belgium; Department of General Internal Medicine, Medical Intensive Care Unit, University Hospitals Leuven, Leuven, Belgium), Nicholas Gilbo (Department of Microbiology, Immunology and Transplantation, Laboratory of Abdominal Transplantation, KU Leuven, Leuven, Belgium; Department of Abdominal Transplant Surgery and Coordination, University Hospitals Leuven, Leuven, Belgium), Ina Jochmans (Department of Microbiology, Immunology and Transplantation, Laboratory of Abdominal Transplantation, KU Leuven, Leuven, Belgium; Department of Abdominal Transplant Surgery and Coordination,
University Hospitals Leuven, Leuven, Belgium), Jacques Pirenne (Department of Microbiology, Immunology and Transplantation, Laboratory of Abdominal Transplantation, KU Leuven, Leuven, Belgium; Department of Abdominal Transplant Surgery and Coordination, University Hospitals Leuven, Leuven, Belgium), Mauricio Sainz Barriga (Department of Microbiology, Immunology and Transplantation, Laboratory of Abdominal Transplantation, KU Leuven, Leuven, Belgium; Department of Abdominal Transplant Surgery and Coordination, University Hospitals Leuven, Leuven, Belgium), Yves Debaveye (Department of Cellular and Molecular Medicine, Laboratory of Intensive Care Medicine, KU Leuven, Leuven, Belgium; Department of Intensive Care Medicine, Intensive Care Unit, Leuven, University Hospitals Leuven, Leuven, Belgium), Jan Gunst (Department of Cellular and Molecular Medicine, Laboratory of Intensive Care Medicine, KU Leuven, Leuven, Belgium; Department of Intensive Care Medicine, Intensive Care Unit, Leuven, University Hospitals Leuven, Leuven, Belgium), Geert Meyfroidt (Department of Cellular and Molecular Medicine, Laboratory of Intensive Care Medicine, KU Leuven, Leuven, Belgium; Department of Intensive Care Medicine, Intensive Care Unit, Leuven, University Hospitals Leuven, Leuven, Belgium), Wim Laleman (Department of Chronic Diseases and Metabolism, Hepatology, KU Leuven, Leuven, Belgium; Department of Gastroenterology and Hepatology, University Hospitals Leuven, Leuven, Belgium), Frederik Nevens (Department of Chronic Diseases and Metabolism, Hepatology, KU Leuven, Leuven, Belgium; Department of Gastroenterology and Hepatology, University Hospitals Leuven, Leuven, Belgium), Hannah Van Malenstein (Department of Chronic Diseases and Metabolism, Hepatology, KU Leuven, Leuven, Belgium; Department of Gastroenterology and Hepatology, University Hospitals Leuven, Leuven, Belgium), Jef Verbeek (Department of Chronic Diseases and Metabolism, Hepatology, KU Leuven, Leuven, Belgium; Department of Gastroenterology and Hepatology, University Hospitals Leuven, Leuven, Belgium), Chris Verslype (Department of Chronic Diseases and Metabolism, Hepatology, KU Leuven, Leuven, Belgium; Department of Gastroenterology and Hepatology, University Hospitals Leuven, Leuven, Belgium), Tania Roskams (Department of Imaging and Pathology, Translational Cell \& Tissue Research, KU Leuven, Leuven, Belgium; Department of Imaging and Pathology, University Hospitals Leuven, Leuven, Belgium), Vincent Vandecaveye (Department of Imaging and Pathology, Translational MRI, KU Leuven, Leuven, Belgium; Department of Radiology, University Hospitals Leuven, Leuven, Belgium), Tim Balthazar (Department of Cardiovascular Diseases, Cardiology, KU Leuven, Leuven, Belgium; Department of Cardiovascular Diseases, Leuven, University Hospitals Leuven, Leuven, Belgium), Christophe Vandenbriele (Department of Cardiovascular Diseases, Cardiology, KU Leuven, Leuven, Belgium; Department of Cardiovascular Diseases, Leuven, University Hospitals Leuven, Leuven, Belgium), Greet Hermans (Department of Cellular and Molecular Medicine, Laboratory of Intensive Care Medicine, KU Leuven, Leuven, Belgium; Department of General Internal Medicine, Medical Intensive Care Unit, Leuven, University Hospitals Leuven, Leuven, Belgium), Peter Rogiers (ZNA Middelheim, Intensive Care Unit, Antwerp, Belgium), Marleen Verhaegen (Anesthesiology and Algology, KU Leuven, Leuven, Belgium; Department of Anesthesiology, University Hospitals Leuven, Leuven, Belgium)

\section{Funding}

Not applicable.

Availability of data and material

Not applicable. 
Code availability

Not applicable.

\section{Declarations}

\section{Conflicts of interest}

The authors declare that they have no conflict of interest.

Ethics approval

Not applicable.

\section{Consent to participate}

Not applicable.

\section{Consent for publication}

Not applicable.

\section{Publisher's Note}

Springer Nature remains neutral with regard to jurisdictional claims in published maps and institutional affiliations.
Received: 3 April 2021 Accepted: 23 May 2021

Published online: 29 June 2021

\section{References}

1. Gupta A, Madhavan MV, Sehgal Ket al (2020) Extrapulmonary manifestations of COVID-19. Nat Med 26(7):1017-1032. https://doi.org/10.1038/ s41591-020-0968-3

2. Martins P, Verdelho MM (2020) Secondary sclerosing cholangitis in critically ill patients: an underdiagnosed entity. GE Port J Gastroenterol 27(2):103-114. https://doi.org/10.1159/000501405

3. Van Aerde N, Van den Berghe G, Wilmer A et al (2020) Intensive care unit acquired muscle weakness in COVID-19 patients. Intensive Care Med. https://doi.org/10.1007/s00134-020-06244-7

4. Gudnason HO, Björnsson ES (2017) Secondary sclerosing cholangitis in critically ill patients: current perspectives. Clin Exp Gastroenterol 10:105-111. https://doi.org/10.2147/CEG.S115518

5. Kaltschmidt B, Fitzek ADE, Schaedler J et al (2021) Hepatic vasculopathy and regenerative responses of the liver in fatal cases of COVID-19. Clin Gastroenterol Hepatol. https://doi.org/10.1016/j.cgh.2021.01.044 